Recherches politiques internationales sur les espaces issus de l'histoire et de la colonisation portugaises

$\mathrm{XV}(2) \mid 2008$

Histoires d'Asie

\title{
Revista Científica, revista de estudos caboverdianos
}

Praia, Universidade de Cabo Verde

\section{Michel Cahen}

\section{OpenEdition}

9 Journals

Édition électronique

URL : http://journals.openedition.org/lusotopie/1572

ISSN : $1768-3084$

Éditeur :

Association des rechercheurs de la revue Lusotopie, Brill, Karthala

Édition imprimée

Date de publication : 20 novembre 2008

Pagination : 287-290

ISSN : 1257-0273

Référence électronique

Michel Cahen, «Revista Científica, revista de estudos caboverdianos », Lusotopie [En ligne], XV(2) | 2008, mis en ligne le 01 février 2016, consulté le 07 mai 2019. URL : http://journals.openedition.org/

lusotopie/1572

Ce document a été généré automatiquement le 7 mai 2019.

Tous droits réservés 


\title{
Revista Científica, revista de estudos caboverdianos
}

\author{
Praia, Universidade de Cabo Verde
}

Michel Cahen

\section{RÉFÉRENCE}

Revista Científica, revista de estudos caboverdianos, Praia, Universidade de Cabo Verde, pas d'ISNN ni d'ISBN.

1 L'université (publique) du Cap-Vert publie désormais régulièrement sa Revista Científica ${ }^{1}$, dont nous rendons compte ici pour les volumes 2 et $3 / 4$. Suite à ce dernier volume, double, il a été annoncé que la revue serait désormais publiée en deux séries distinctes, de sciences sociales et humaines d'une part, et de sciences expérimentales, exactes et du vivant d'autre part. Les articles sont précédés d'un résumé dans les deux langues autres que celle de l'article (portugais : résumés en anglais et français, etc.).

2 旍 Dans la Revista Científica, 2, janvier 2006, 204 p., on notera en particulier, du point de vue de l'analyse politique :

3 - António Germano LIMA, « A cultura material como fonte histórica : o caso do património construido da Praia » : 7-26. L'histoire locale comme contribution à l'histoire matérielle globale ; de nombreuses données sur l'histoire de Praia.

4 - João Lopes FILHo, "O multiculturalismo e a integração dos filhos dos imigrantes capverdianos »: 27-57. Les pratiques d'enseignement multiculturel favorisent la stabilisation des jeunes de «seconde génération » dans ce pays d'accueil. L'article aborde la situation des jeunes Capverdiens au Portugal ; il est d'ailleurs dommage qu'il n'ait pas étendu la réflexion à d'autres pays, car il n'est pas sûr que le Portugal soit, du fait de sa proximité culturelle, le pays le plus typique de ces problématiques.

5 - Kesha FIKES, "The Cape Verdean Woman Worker and Portuguese Civil Society, 1965-1982 » : 59-69. Sous le colonialisme tardif (1961-1974), la présence de Capverdiens au 
Portugal concernait surtout les hommes; cependant, à la fin de la période, le nombre de femmes s'est accru. Mais elles n'étaient pas censées travailler : ceci se fit cependant au tournant des années 1970, avec l'apport de dignité et conscience que donne toujours un travail extérieur à la maison. Mais ensuite, dans le Portugal européen et néolibéral, les femmes capverdiennes sont entrées dans la problématique de la féminisation de certaines industries cherchant à préserver de bas salaires. Mais la part du travail comme femmes de ménage, etc. reste considérable et les femmes capverdiennes rencontrent sur ce plan les femmes portugaises pauvres.

6 - Odair Bartolomeu VARELA, « Cabo Verde : um desafio teórico-paradigmático ou um caso singular?» : 71-88. Les Capverdiens n'ont pas fini de réfléchir à l'exceptionnalité de leur archipel! Serait-ce - aussi - un héritage colonial issu de l'exceptionnalité portugaise? Toujours est-il que, selon les théories des «États fragiles ", le Cap-Vert réunirait toutes les conditions (économiques, géographiques, écologiques) pour en faire partie. Or il n'en est rien. l'auteur examine l'histoire de cette trajectoire singulière et l'avantage que fut pour le pays le fait que c'est, en pratique, la direction politique du PAIGC qui y prit le pouvoir, pendant que la direction militaire le prenait en Guinée-Bissau. la «capacité politique " aura été un avantage certain dans la construction de l'État et un positionnement habile évitant les clivages de la Guerre froide. L'avenir n'est cependant pas assuré, d'autant plus que l'hétérogénéité de la CPLP l'empêche d'être un acteur actif sur la scène internationale.

7 - João ESTEVÃo, «Desenvolvimento económico e mudança institutional: o papel do Estado » : 89-108. Les économistes classiques des années soixante ont toujours considéré que l'accumulation $\mathrm{du}$ capital était une question centrale pour les pays en développement. La "main invisible» n'était plus tant celle du marché que de l'État régulateur, en une posture keynésienne. Dans les années soixante-dix, ces thèses furent écartées au profit de celles sur l'efficience de l'affectation des ressources. Le néoinstitutionnalisme à son tour critiqua ces théories dans la décennies 1990, mixant une partie des thèses du vieux «classicisme » et du vieux «structuralisme ». Cet article, en portugais, rendra à n'en pas douter service aux étudiants capverdiens, mais il est dommage que l'auteur mais pas abordé les conséquences de ces évolutions pour le CapVert. Cela nous aurait intéressés, nous, les Non-Capverdiens !

8 - António dos Reis DUARTE, "Conceito e mensuração da pobreza: diferentes abordagens »: 109-122. Comment tomber d'accord sur une définition commune du concept de pauvreté ? L'auteur aborde, avec force mathématique, la question de la distinction que doit recouvrer ce concept dans les pays riches et les pays en développement.

9 - Milton Nascimento de Sena PAIVA, «La réception du droit international dans l'ordre juridique interne capverdien: le dispositif constitutionnel»: 123-136. Quelle est la technique d'insertion du droit international adoptée par la Constitution capverdienne? Cette dernière est fortement inspirée du droit portugais, mais les études jurisprudentielles furent surtout marquées par le droit français. Pourtant, la réception du droit international s'y fait sans transformation en norme interne. Mais la vérification de la constitutionnalité des traités internationaux signés se fait, grosso modo, de la même manière que dans les constitutions portugaise et française. 
10 - Manuel Brito SEMEDO, « A emigração na formação da elite letrada cabo-verdiana (séc. XIX e xx)» : 137-142. L'article insiste en particulier sur le rôle de l'émigration aux États-Unis et l'activité de l'Église pentecôtiste nazaréenne.

11 - Margarida FRAGoso, « Universidades públicas em pequenos Estados insulares » : 159-178. Le Cap-Vert n'est pas abordé par cet article, mais la volonté comparatiste est évidente : comment l'université capverdienne en formation peut-elle apprendre des exemples de Chypre, Malte, Maurice, Pacifique Sud (Îles Cook, etc.), Indes occidentales (Jamaïque, etc.) ? Ces universités cherchent à être à la pointe des techniques informatiques afin de rompre leur isolement, et donnent la priorité à la Recherche-Développement. La diversification des financements se fait notamment par la consultance.

12 Diverses recensions critiques d'ouvrages terminent cette livraison particulièrement stimulante.

13 或 Dans la Revista Científica, 3/4, novembre 2006, 292 p., les articles sont désormais classés en « Sciences sociales et humaines », « Sciences de la Vie », « Énergie alternatives » et un dossier "Éducation ». Dans les " Ciências sociais e humanas », on notera en particulier :

14 - Nicolas QUINT, «African words and calques in Capverdean Creole (Santiago dialect)»: 9-42. Dans cet article en anglais publié par le chercheur français dans une revue capverdienne, c'est la place des mots d'origine africaine dans le créole contemporain qui est étudiée. L'article est essentiellement linguistique, mais aborde toutefois la question du lien entre persistance (ou non) de traces culturelles africaines par le biais de la survie de mots. Utile bibliographie (39-41).

15 - João Lopes FilHo, "O padre António Vieira e Cabo Verde » : 43-63. L'article étudie les quelques textes du célèbre prêcheur du XVII ${ }^{e}$ siècle écrits à l'occasion d'un bref séjour qu'il fit au Cap-Vert.

16 - António Germano LIMA, «Eugénio Tavares: contribuição a investigação históricocultural da sociedade cabo-verdeana »:65-83. L'article porte notamment sur les aspects sociopolitiques de l'œuvre du journaliste, écrivain, poète et compositeur de la fin du XIX et début du $\mathrm{xx}^{\mathrm{e}}$ siècles. Voir aussi le site <www.eugeniotavares.org/>, souvent cité dans l'article.

17 La section « Energias alternativas » présente des études très techniques (connaissiez-vous le TWSPP, pour thermal wind solar power plant, un nouveau système qui pourrait être intéressant pour l'archipel ?) ou de cas étrangers (Islande).

Dans le dossier « Educação », on trouve notamment :

19 - Arlindo VIEIRA, «Análise de necessidades na formação de professores do Ensino Básico Integrado (EBI) em Cabo Verde. Contributo para o seu estudo » : 139-156. L'enquête est fondée sur un échantillon de 200 intervenants (majoritairement instituteurs). Il ne semble pas que la méthode quantitative ait apporté des révélations sur leurs aspirations...

20 - Amália de Melo LOPES, « $O$ ensaio da leitura no ensino secundário » : 157-170. L'article aborde les $9^{\mathrm{e}}$ et $10^{\mathrm{e}}$ années (équivalent des Troisièmes et Secondes françaises), exclusivement pour la langue portugaise (le créole n'est pas abordé). L'étude montre l'inadéquation des programmes avec le rapport à la lecture portugaise qu'ont les élèves de ce niveau et le blocage qui s'en suit pour les niveaux supérieurs.

21 - Paulina Varela tAVARES, «Educação (capital humano) como instrumento de desenvolvimento económico e social de Cabo Verde: desafios competitivos e a importância da Universidade pública»: 183-199. Dans cet article tiré d'une tese de 
mestrado soutenue au Brésil, l'auteure défend en particulier la nécessité d'approfondir les synergies entre les tâches du développement et les fonctions de la nouvelle université publique.

- Lionildo Sá NOGUEIRA, « A utilisação das novas tecnologias de informação e comunicação no ensino secundário. $O$ caso de Cabo Verde »: 201-214. Cet article contient de très intéressantes statistiques sur l'usage des ordinateurs par les professeurs du secondaire et illustre les grandes inégalités de situation selon les îles et types de lycée.

- Mária Luísa Soares INOCÊNCIO, « Formação a distância de professores do EBI em exercício de funções : contribuição para sua discussão » : 215-229. L’article aborde la nécessité, et la difficulté de la formation à distance pour les instituteurs.

24 - Ana Cristina Pires FERREIRA, « La gestion du système éducatif capverdien : quel(s) logique (s) ?» [sic] : 231-243.L'article présente une analyse de la gestion de l'éducation de 1991 à 2004, c'est-à-dire en temps de grandes réformes. Comment « piloter » des projets dans un système soumis à de fortes contraintes, et de manière à ce que le " pilotage " ne soit pas un «contrôle total»? L'article présente en quelque sorte un bilan d'étape : nécessité d'une politique centralisée pour la massification de l'éducation, mais nécessité aussi de gestion participative et de décentralisation.

25 - Silvia Castro CARDOSo, « Currículo : percursos, processos e actores » : 245-261. Discussion (souvent par trop technique) des procédés de définition des programmes et du contenu de l'enseignement.

Ce gros volume s'achève par quelques recensions ( $c f$. notamment la recension de Zelinda Cohen sur le livre de Maria José Lopes, Surgimento de Câmaras municipais nas Ilhas do Norte, Praia, Instituto do Arquivo Histórico Nacional, 2005: 265-268) et des nouvelles scientifiques et institutionnelles, comme les Projectos de Investigação Pró-África-Cabo Verde, financés par le Brésil (p. 281).

27 Contact: Universidade de Cabo Verde, CP 379-C, Praia (Santiago), République des Îles du Cap-Vert, fax : 00238261 26 60, courriel : < cni@gov1.gov. cv >, site : < www.cniunicv.cv >. Juin 2007

\section{NOTES}

1. Voir le vol. XV (1) de Lusotopie pour les volumes 0 et 1. 